\title{
Dopamine transporter imaging versus myocardial MIBG scintigraphy for the diagnosis of prodromal synucleinopathies_-and other updates on autonomic research
}

\author{
Mitchell G. Miglis ${ }^{1} \cdot$ Srikanth Muppidi ${ }^{1}$
}

Received: 26 February 2019 / Accepted: 27 February 2019 / Published online: 23 March 2019

(c) Springer-Verlag GmbH Germany, part of Springer Nature 2019

Keywords Dopamine transporter scan · Cardiac sympathetic imaging · Hereditary sensory and autonomic neuropathies . Parkinson disease $\cdot$ Pure autonomic failure

\section{The brain and the heart revisited: DAT scan versus myocardial MIBG scanning}

In recent years, several promising imaging techniques have emerged in the evaluation of patients with suspected $\alpha$-synucleinopathies. These tools can be especially helpful in the prodromal stages of these conditions, when diagnosis remains challenging and patients are struggling to accept several potential outcomes. For example, will a diagnosis of rapid eye movement (REM) sleep behavior disorder (RBD) transform into the fatally aggressive multiple system atrophy (MSA) or the less aggressive Parkinson's disease (PD)? Will a diagnosis of pure autonomic failure (PAF) remain so, or transform into dementia with Lewy bodies (DLB)? Longitudinal natural history studies are currently being carried out to better answer this question [1], but these studies take time.

Currently, there is no single biomarker with perfect sensitivity and specificity, and most clinicians use a combination of patient history, physical examination, autonomic and sleep data, and imaging techniques, all filtered through the lens of clinical judgement to eventually reach a diagnosis, which often remains uncertain. ${ }^{123}$ I-ioflupane dopamine transporter (DAT) imaging and ${ }^{23}$ I-metaiodobenzylguanidine (MIBG) myocardial scintigraphy are two such imaging modalities used in these evaluations, although longitudinal data on their usefulness in practice is limited.

In a manuscript published in the October 152018 issue of Movement Disorders Clinical Practice [2], 18 patients

Srikanth Muppidi

muppidis@stanford.edu

1 Stanford Neurosciences Health Center, 213 Quarry Road, 2nd Floor, Palo Alto, CA 94304, USA with prodromal synucleinopathies were studied at baseline and at longitudinal follow-up. Inclusion criteria were either: (1) autonomic impairment, defined as orthostatic hypotension (which they classified as PAF), constipation, or bladder dysfunction; (2) RBD; or (3) mild cognitive impairment or psychiatric symptoms (the authors do not define which psychiatric symptoms specifically). Patients with multiple system atrophy (MSA) in whom cardiac MIBG scan is supposed to be normal were excluded, though it is not clear how exactly this was assessed.

The authors performed a standard neurological examination and cognitive testing (Mini-Mental State Exam) in all patients. Cardiovascular autonomic reflex testing and polysomnography were performed when clinically indicated at the discretion of the physicians. Some patients had cerebrospinal fluid collection to measure tau, $\beta$-amyloid, and $\alpha$-synuclein levels. All patients had a DAT scan and a cardiac MIBG scan performed at their initial visit. Olfactory testing was not performed.

The mean age of the patients was 75.5 years (range 67-86 years), and most had a long history of symptoms (mean duration of disease 14.5 years, range 1-37 years) and the majority were men. The neurological diagnoses included constipation/RBD in $10(56 \%)$, constipation/RBD/PAF in $6(33 \%)$, and constipation/PAF in the remaining 2 patients (11\%). Thus, $16 / 18$ (89\%) of this cohort had RBD. Of the 18 patients, 5 eventually developed PD, one developed DLB, and 1 developed mild cognitive impairment. Thus, 11 remained with either PAF or RBD. Among the 18 patients, all but one (94\%) had reduced myocardial intake on MIBG scan, whereas only $56 \%$ had reduced dopamine transporter on DAT scan, indicating, according to the authors, "a much greater sensitivity of MIBG for detecting prodromal $\alpha$-synunclein disease." 
The study had significant limitations, including the small sample size, no control group, lack of consistent autonomic and sleep testing, and lack of standardized neurological/ motor (e.g., UPDRS) rating scales. Olfactory testing was not performed either. The largest potential limitation, however, is the fact that most patients had RBD. It has now been demonstrated that almost all patients with RBD, even those with clinically isolated RBD (without CNS manifestations), have evidence of postganglionic cardiac sympathetic denervation on MIBG scanning [3]. In fact, a diagnosis of RBD is more of a predictor of an abnormal MIBG scan than a diagnosis of PD. Thus, there is something unique to RBD that favors postganglionic $\alpha$-synuclein deposition, as well as deposition in the pons. This study indicates that MIBG is likely more sensitive at detecting abnormalities in the premotor or prodromal phase of the $\alpha$-synucleinopathies, but is the specificity good enough to help distinguish which condition patients will eventually develop? This is the important question, and, as always, future studies are needed. Also, the fact that MIBG scanning is not widespread in the U.S. makes the generalizability of these results in North American cohorts quite challenging.

\section{Moving beyond diagnosis: treatment for hereditary sensory autonomic neuropathies}

The hereditary sensory autonomic neuropathies (HSAN) are rare genetic neuropathies with sensory and autonomic manifestations [4]. HSAN type 1 (HSAN1) is an adult-onset autosomal dominant progressive form that presents with sensory loss followed by pain and other positive sensory symptoms. Because of their sensory loss, patients are prone to develop ulcers and sometimes osteomyelitis, ultimately leading to distal amputation. HSAN1 is caused by mutations in the SPTLC1 and SPTLC2 genes, both encoding for the enzyme serine palmitoyltransferase, which is critical for the synthesis of sphingolipids as it catalyzes the conjugation of palmitoyl-CoA with serine. Mutations in the SPTLC genes shift the serine palmitoyltransferase enzyme affinity from serine towards L-alanine, resulting in the synthesis of 1-deoxysphingolipids, which are are neurotoxic to peripheral nerves. Interestingly, oral L-serine therapy reduces 1-deoxysphingolipid levels in patients with HSAN1, as a previous small open-label study demonstrated [5].

The January 222019 issue of Neurology includes the results of a randomized placebo-controlled trial of L-serine in patients with HSAN1 followed by a 1-year open-label treatment phase [6]. They recruited 18 patients randomized to L-serine $(400 \mathrm{mg} / \mathrm{kg} / \mathrm{day})$ given in 3 doses or matching placebo. The primary outcome measure was the Charcot-Marie-Tooth Neuropathy Score version 2 (CMTNS). Multiple secondary outcome measures were analyzed, including nerve conduction studies, epidermal nerve fiber density, and patient reported measures. Additionally, to assess the biological basis, researchers also measured plasma sphingolipid levels in patients.

During the randomized placebo-controlled phase of the study (year 1), there were no dropouts. Patients on L-serine showed significant improvements in the CMTNS compared to the placebo group ( -1.5 units, $95 \% \mathrm{CI}-2.8$ to $-0.1, P=0.03$ ). Additionally, in the $\mathrm{L}$-serine group, there was a trend towards improvement in distal epidermal fiber density, assayed from 3-mm punch skin biopsies at the distal leg, and towards improvement in autonomic function as measured by a lower composite autonomic severity score (CASS), although none of these differences were statistically significant. There was no clear improvement in pain. There was also no difference in serum L-serine level between groups, although 1-deoxysphingolipid levels were indeed lower in the active agent group. There were no severe adverse effects, and only 2 patients dropped out of the study during the open-label extension phase for reasons unrelated to disease or treatment.

These exciting findings for a progressive neuropathy with severe peripheral nerve manifestations and, until now, no treatment are promising. Conversely, the clinical benefit seems to be relatively modest. This might be because patients in this study already had advanced disease with a disease duration of more than 2 decades, making a reversal in their disease progression unlikely. Additionally, the CMTNS was originally developed for patients with Charcot-Marie-Tooth disease without significant small fiber neuropathy manifestations, as seen in HSAN1, and it is unclear if this measure is valid or sensitive enough to detect subtle changes in small fiber function. Nerve physiology data such as nerve conduction studies and CASS are not likely to be sensitive enough to detect minor improvements in chronic neuropathies. Finally, there was an improvement in distal nerve fiber density, but not proximal nerve fiber density, the exact opposite of the expected results of treatment of length-dependent neuropathies, and it is unclear if this was due to variability in skin biopsy technique or if there is an underlying true biological basis for this finding. In spite of these limitations, this small study represents a commendable initial step towards developing further therapies for patients with rare genetic diseases with autonomic involvement.

Funding None.

\section{Compliance with ethical standards}

Conflict of interest None. 


\section{References}

1. Kaufmann H, Norcliffe-Kaufmann L, Palma J-A, Biaggioni I, Low PA, Singer W et al (2017) The natural history of pure autonomic failure: a U.S. prospective cohort. Ann Neurol 81:287-297. https ://doi.org/10.1002/ana.24877.

2. Sakakibara R, Tateno F, Aiba Y, Ogata T, Kishi M, Terada H et al (2019) MIBG myocardial scintigraphy identifies premotor PD/DLB during a negative DAT scan period: second report. Mov Disord Clin Pract 6:46-50. https://doi.org/10.1002/mdc3.12697

3. Miyamoto T, Miyamoto M, Suzuki K, Nishibayashi M, Iwanami M, Hirata K (2008) 123I-MIBG cardiac scintigraphy provides clues to the underlying neurodegenerative disorder in idiopathic REM sleep behavior disorder. Sleep 31:717-723
4. Auer-Grumbach M (2013) Hereditary sensory and autonomic neuropathies. Handb Clin Neurol 115:893-906. https://doi. org/10.1016/B978-0-444-52902-2.00050-3

5. Garofalo K, Penno A, Schmidt BP, Lee H-J, Frosch MP, von Eckardstein A et al (2011) Oral L-serine supplementation reduces production of neurotoxic deoxysphingolipids in mice and humans with hereditary sensory autonomic neuropathy type 1 . J Clin Invest 121:4735-4745. https://doi.org/10.1172/JCI57549

6. Fridman V, Suriyanarayanan S, Novak P, David W, Macklin EA, McKenna-Yasek D et al (2019) Randomized trial of L-serine in patients with hereditary sensory and autonomic neuropathy type 1. Neurology 92:e359-e370. https://doi.org/10.1212/WNL.00000 00000006811 